Med Klin Intensivmed Notfmed 2022 · 117:457-458 https://doi.org/10.1007/s00063-021-00874-5 Online publiziert: 4. Oktober 2021

○ Der/die Autor(en) 2021

\section{Erratum zu: Mit dem Rettungsdienst direkt in die Arztpraxis - eine wirkungsvolle Entlastung der Notaufnahmen?}

Tobias Lindner' ${ }^{1}$ Alessandro Campione ${ }^{2} \cdot$ Martin Möckel $^{1}$. Cornelia Henschke² . Janosch Dahmen ${ }^{3,4} \cdot$ Anna Slagman'

${ }^{1}$ Notfall- und Akutmedizin, Charité - Universitätsmedizin Berlin Campus Virchow-Klinikum und Campus Mitte, Berlin, Deutschland

${ }^{2}$ Fachgebiet Management im Gesundheitswesen, Technische Universität Berlin, Berlin, Deutschland ${ }^{3}$ Fakultät für Gesundheit, Department Humanmedizin, Universität Witten/Herdecke, Witten, Deutschland ${ }^{4}$ Ärztliche Leitung Rettungsdienst, Berliner Feuerwehr, Berlin, Deutschland

\section{Erratum zu:}

Med Klin Intensivmed Notfmed 2021

https://doi.org/10.1007/s00063-02100860-x

In diesem Artikel wurde die Abb. 4 falsch wiedergegeben. Die Abbildung hätte wie hier dargestellt erscheinen müssen. Der Originalbeitrag wurde korrigiert.

\section{Korrespondenzadresse}

Dr. med. Tobias Lindner

Notfall- und Akutmedizin, Charité -

Universitätsmedizin Berlin Campus Virchow-

Klinikum und Campus Mitte

Augustenburger Platz 1, 13353 Berlin,

Deutschland

tobias.lindner@charite.de

Funding. Open Access funding enabled and organized by Projekt DEAL.

Open Access. Dieser Artikel wird unter der Creative Commons Namensnennung 4.0 International Lizenz veröffentlicht, welche die Nutzung, Vervielfältigung, Bearbeitung, Verbreitung und Wiedergabe in jeglichem Medium und Format erlaubt, sofern Sie den/die ursprünglichen Autor(en) und die Quelle ordnungsgemäß nennen, einen Link zur Creative Commons Lizenz beifügen und angeben, ob Änderungen vorgenommen wurden.

Die in diesem Artikel enthaltenen Bilder und sonstiges Drittmaterial unterliegen ebenfalls der genannten Creative Commons Lizenz, sofern sich aus der Abbildungslegende nichts anderes ergibt. Sofern das betreffende Material nicht unter der genannten Creative Commons Lizenz steht und die betreffende Handlung nicht nach gesetzlichen Vorschriften erlaubt ist, ist für die oben aufgeführten Weiterverwendungen des $\mathrm{Ma}$ terials die Einwilligung des jeweiligen Rechteinhabers einzuholen.

Weitere Details zur Lizenz entnehmen Sie bitte der Lizenzinformation auf http://creativecommons.org/ licenses/by/4.0/deed.de.

Die Online-Version des Originalartikels ist unter https://doi.org/10.1007/s00063-021-00860-x zu finden.

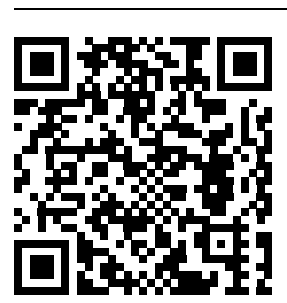

QR-Code scannen \& Beitrag online lesen 


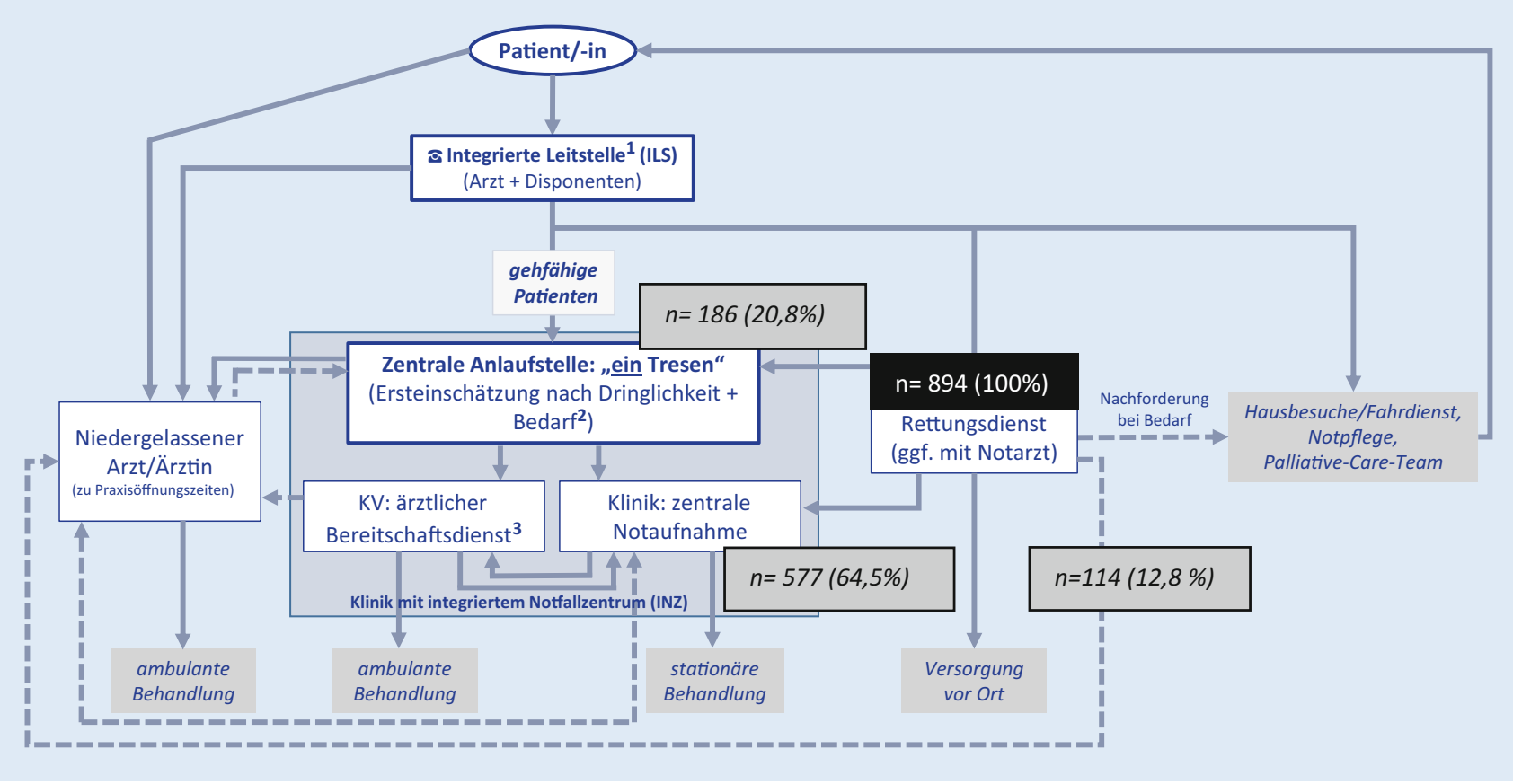

Abb. 4 \ Skizze der integrierten Notfallversorgung nach dem Entwurf des Sachverständigenrats mit ergänzender Darstellung einer möglichen Patientenverteilung durch den Rettungsdienst unter der Annahme, dass 1.) alle "dringlichen“ bzw. „weniger dringlichen“ Fälle mit stationärer Weiterbehandlung direkt in die zentrale Notaufnahme, 2.) alle „weniger dringlichen" Fälle mit ambulanter Weiterbehandlung im Zeitraum Montag bis Freitag, 08.00 bis $19.00 \mathrm{Uhr}$ (ohne Feiertage) zum niedergelassenen Arzt (in die Praxis) bzw. 3.) außerhalb dieser Zeiten an eine "zentrale Anlaufstelle“ disponiert werden. Medizinisch-inhaltliche Aspekte bleiben bei der Zuordnung unberücksichtigt. 'Nutzt Notfallalgorithmen/Versorgungspfade, Zugriff auf verfügbare Ressourcen, bucht Termine bei zentraler Anlaufstelle. ${ }^{2}$ Ersteinschätzung möglichst durch ärztliche "Generalisten", breit weitergebildete (Allgemein-)Mediziner mit notfallmedizinischer Erfahrung. Die Ersteinschätzung muss weisungsunabhängig vom Krankenhaus erfolgen. ${ }^{3} \mathrm{Ggf}$. mit kinder- und/oder augenärztlichem Bereitschaftsdienst und/oder psychiatrischem Kriseninterventionsdienst. (Die Option „Versorgung vor Ort“ wurde nicht berücksichtigt. 17 „weniger dringliche" Fälle mit ambulanter Weiterbehandlung konnten zeitlich nicht zugeordnet werden). (Aus [4]. Mit freundl. Genehmigung (c) Referat 515 - Geschäftsstelle des Sachverständigenrats zur Begutachtung der Entwicklung im Gesundheitswesen [SVR], Referat „Ethik im Gesundheitswesen, Sachverständigenrat Gesundheit“, Bundesministerium für Gesundheit. Alle Rechte vorbehalten) 